\title{
The predictive value of vascular endothelial growth factor-A gene polymorphism for clinical outcomes among acute ST-segment elevation myocardial infarction patients: A single center prospective study
}

\author{
Inna M. Kutia, Mykola P. Kopytsya, Yaroslava V. Hilova, Olga V. Petyunina, Alexander E. Berezin*(®)
}

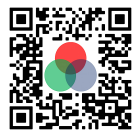

Use your smartphone to scan this QR code and download this article

L.T.Malaya Therapy National Institute NAMSU, 2 A Liubovi Maloy av., 61039, Kharkiv, Ukraine

\section{Correspondence}

Alexander E. Berezin, L.T.Malaya Therapy National Institute NAMSU, 2A Liubovi Maloy av., 61039, Kharkiv, Ukraine.

Email: aeberezin@gmail.com; dr_berezin@mail.ru

History

- Received: Mar 12, 2020

- Accepted: May 02, 2020

- Published: May 25, 2020

DOI : 10.15419/bmrat.v7i5.602

\section{Check for updates}

\section{Copyright}

(C) Biomedpress. This is an openaccess article distributed under the terms of the Creative Commons Attribution 4.0 International license.

\begin{abstract}
Background: Vascular endothelial growth factor (VEGF) is an angiopoetic factor; its variability in circulating levels is mediated by expression of specific VEGF-A gene variants. The aim of this study was to investigate the predictive role of VEGF-A gene polymorphism in clinical outcomes of STelevation myocardial infarction (STEMI) patients. Methods: For the study, 135 patients with acute STEMI and 30 healthy volunteers were enrolled. The G634C polymorphism in VEGF-A gene was performed by real-time polymerase chain reaction at baseline. The 6-month combined clinical endpoint was then determined. Design: The study was an open prospective single-center cohort study. Results: The entire patient population was distributed into two groups based on the G634G-genotype ( $n=70$ ) and combination of G634C and C634C-genotypes ( $n=65)$. Unadjusted multivariate regressive logistic analysis showed peak troponin I levels at admission, Killip class of heart failure $>2$, GC/CC polymorphisms in VEGF-A gene, and dynamic increase of NT-pro brain natriuretic peptide (BNP) and VEGF-A levels for 6 months, which were independent predictors for the combined clinical endpoint. After adjustment for dynamic changes of NT-proBNP and VEGF-A levels, we found that GC/CC polymorphisms in the VEGF-A gene was an independent predictor of clinical outcome. Kaplan-Meier curves demonstrated that STEMI patients with GG VEGF-A genotype had a lower frequency of clinical combined endpoint accumulation when compared to those who had GC/CC VEGF-A genotypes (Log-rank $p=0.02$ ). Conclusion: The G634C polymorphism in the VEGF-A gene was found to be an independent predictor for 6 -month clinical combined endpoint in STEMI patients.

Key words: ST-segment elevation myocardial infarction, single nucleotide polymorphism G634C, vascular endothelial growth factor, prediction, outcomes
\end{abstract}

\section{INTRODUCTION}

Patients with acute ST-elevation myocardial infarction (STEMI) undergoing successful primary percutaneous coronary intervention (PCI) yield significant differences with respect to in-hospital mortality, hospital length of stay, cardiovascular (CV) events and complications, and late survival ${ }^{1,2}$. Previous systematic network meta-analysis has shown that multivessel coronary artery disease (CAD) in STEMI patients is a much more powerful trigger than singlevessel CAD for survival and prognosis, regardless of revascularization strategy ${ }^{3}$. Additionally, complete revascularization based on comprehensive condition is disputed as the most suitable choice in actual clinical situations such as hemodynamic instability, newly onset heart failure, and high risk of severe short-term $\mathrm{CV}$ complications ${ }^{4,5}$. However, staged revascularization strategy is recommended for complex non- infarct-related artery lesions under evaluation (fractional flow reserve, intravascular ultrasound, and optical coherent tomography) ${ }^{6,7}$. In fact, long-term risk of $\mathrm{CV}$ events and severity of left ventricular post-MI remodeling remain to be uncertain and unpredictable following successful PCI with TIMI-III restoration of blood flow through culprit artery and even after complete revascularization ${ }^{8,9}$. As a result, the complexity of STEMI patients undergoing primary successful PCI has not changed significantly.

Recent studies have revealed that effective angiogenesis and neovascularization play crucial roles in early restoration of microvascular perfusion in damaged myocardium and in prevention of late left ventricular remodeling for months after reperfused STEMI ${ }^{10-12}$. Vascular endothelial growth factor A (VEGF-A) is a key regulator of angiogenesis which mediates proangiopoetic, anti-inflammatory and anti-oxidative

Cite this article : Kutia I M, Kopytsya M P, Hilova Y V, Petyunina O V, Berezin A E. The predictive value of vascular endothelial growth factor-A gene polymorphism for clinical outcomes among acute STsegment elevation myocardial infarction patients: A single center prospective study. Biomed. Res. Ther.; 7(5):3744-3759. 
capacities by acting via appropriate receptors ${ }^{13,14}$. VEGF-A synthesis and secretion are mediated by hypoxia through overexpressed hypoxia-induced factor1 (HIF-1) and nuclear factor-kappa B (NF-kB) ${ }^{14}$. VEGF-A contributes to the development of collaterals by increasing vascular permeability, stimulating proliferation and migration of progenitor and mature endothelial cells, inhibiting apoptosis of endothelial precursors, inducing matrix metalloproteinases, and activating von Willebrand factor ${ }^{15}$.

Previous studies have shown that the levels of VEGFA in patients with acute STEMI have been found to be significantly higher than those of healthy volunteers ${ }^{16}$. After PCI, the concentration of biomarkers were seen to dramatically decrease by approximately $70 \%$, with the most profound decline seen in individuals with the highest initial VEGF-A concentrations ${ }^{17}$. There is strong evidence regarding the negative predictive value of lowered circulating levels of VEGF-A and major cardiovascular events (MACEs) after STEMI in a one-year observation ${ }^{18}$. Interestingly, the circulating levels of VEGF-A showed profound variability in healthy volunteers as well as in patients with STEMI; these results were associated with a functional G634C (rs 2010963) polymorphism in the VEGF-A gene ${ }^{19,20}$.

We hypothesized that the functional variant of the VEGF-A gene can determine dynamic changes of VEGF-A levels after PCI and potentiate the endogenous repair system activity to protect damaged myocardium, after STEMI, from microvascular obstruction and thereby improving long-term prognosis. The aim of this study was to investigate the predictive role of the VEGF-A gene polymorphism (rs 2010963) in clinical outcomes of STEMI patients after complete successful PCI.

\section{MATERIAL AND METHODS}

\section{Patient population}

A total of 190 patients with confirmed acute STEMI were evaluated for participation in the study (Figure 1). From the entire population of STEMI $(\mathrm{n}=190)$, according to inclusion and non-inclusion criteria we enrolled 140 individuals with acute STEMI who were admitted to intensive care unit of "L.T.Malaya TNI NAMSU" (Kharkiv, Ukraine) within a given period from 2016 January to 2019 June. Acute STEMI was diagnosed according to the European Cardiology Society (ECS) Guidelines $(2017)^{21}$. Thirty healthy volunteers were enrolled as a control group.

Inclusion criteria included: established acute STEMI, age $>18$ years old, lack of contraindications to PCI, and written informed consent to participate in the study. Exclusion criteria included: previous myocardial infarction, established chronic heart failure, severe comorbidities (anemia, chronic obstructive lung disease, bronchial asthma, liver cirrhosis, chronic kidney disease with declined glomerular filtration rate $<$ $35 \mathrm{~mL} / \mathrm{min} \times 1.73 \mathrm{~m}^{2}$, valvular heart disease, bleeding, etc.), known malignancy and pregnancy, and/or inability to understand or provide informed consent. Primary PCI with bare-metal stent (COMMANDER, "Alvimedica", Turkey) implantation was performed in 104 patients within 6-12 hours after initial acute STEMI confirmation in the V.T. Zaytsev Institute of General and Emergency Surgery NAMSU (Kharkiv, Ukraine). Systemic thrombolysis (tPA tenecteplase i.v. bolus per conventional protocol) was carried out in 31 STEMI patients prior to PCI. All acute STEMI patients received adjuvant treatment due to current ESC recommendations ${ }^{21}$. TIMI III blood flow restoring through culprit artery was determined for every reperfused patient with acute STEMI (Figure 2).

\section{Ethical declaration}

All procedures in the study involving human participants were performed in accordance with ethical standards according to the 1964 Helsinki declaration and its later amendments, or comparable ethical standards approved by the local ethics committee (Protocol №6, 30.05.2017). Written voluntary inform consent was obtained from each patient before entering the study.

\section{Sample size calculation}

The sample size was calculated through the effect size estimation (0.99), the type of present study, providing study power of $80 \%$ and type I error 5\%, STEMI inhospital mortality of $7.5 \%$, and one-year mortality of $14 \%^{22}$. The sample size was 135 individuals.

\section{Coronary angiography}

Conventional coronary angiography was performed immediately after admission of the patients to the hospital using Digital X-Ray system "Integris Allura" (Philips Healthcare, Best, The Netherlands), and managed by radial or femoral vascular access. Coronary arteries were visualized with two-to-three orthogonal projections per conventional protocol. The number of views obtained was decided by the operator depending on coronary anatomy. The main coronary arteries were left main coronary artery, left anterior descending branch, left circumflex branch, right main coronary artery, and right coronary descending branch. In this study, the contrast "Ultravist-370" 


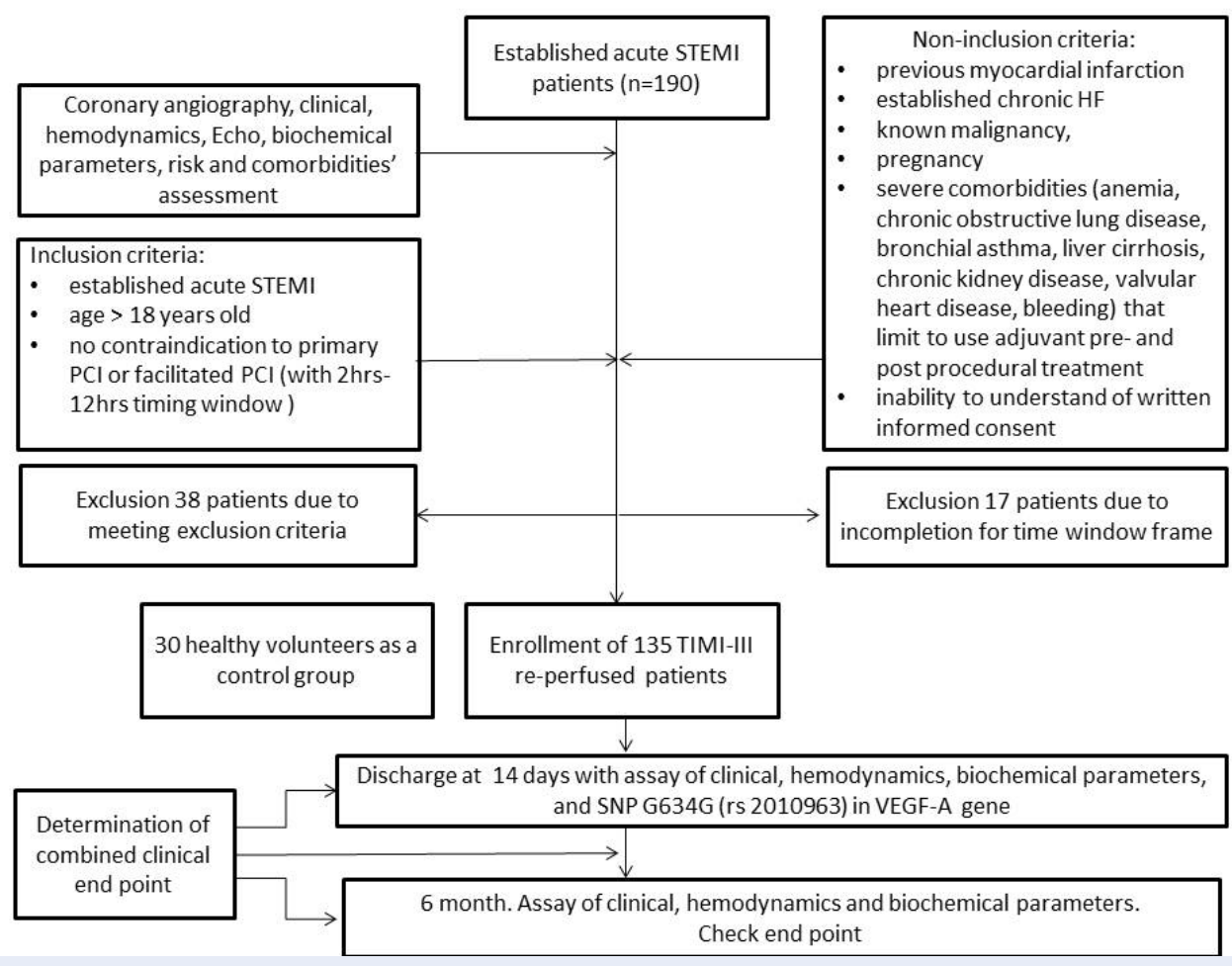

Figure 1: Study design flow chart.

(Baier Pharma GmbH, Germany) and automatic contrast injector were used. The contrast amount used in coronary angiography in each injection was $8-10$ $\mathrm{mL}$ at $4 \mathrm{~mL} / \mathrm{s}$ for the left coronary artery and $6 \mathrm{~mL}$ at $3 \mathrm{~mL} / \mathrm{s}$ for the right coronary artery (radiation exposure 20 to $35 \mathrm{mGycm}$ ). After coronary angiography, two experienced interventional cardiologists discussed the captures and filled in the final report of the results of the procedure after reaching consensus.

\section{Determination of risk factors and comor- bidities}

Hypercholesterolemia (HCE) was diagnosed if total cholesterol (TC) level was $>5.2 \mathrm{mmol} / \mathrm{L}$, and/or low density lipoprotein cholesterol (LDL) level was $>3.0$ $\mathrm{mmol} / \mathrm{L}$, and/or level of triglycerides $(\mathrm{G})$ was $>1.7$ $\mathrm{mmol} / \mathrm{L}$, according to the ECS dyslipidemia guideline $(2016)^{23}$. Hypertension was diagnosed if systolic blood pressure (SBP) was $>140 \mathrm{~mm} \mathrm{Hg}$ and/or diastolic blood pressure (DBP) $>90 \mathrm{~mm} \mathrm{Hg}$, according to the European guideline on diagnostics and treatment of arterial hypertension (2018) ${ }^{24}$. Heart failure was diagnosed according to the ESC guidelines for the diagnosis and treatment of acute and chronic heart failure $(2016)^{25}$. Positive smoking history was defined as having smoked daily or occasionally in the past.

\section{Transthoracic Echocardiography and Doppler}

Transthoracic echocardiography was performed on "Aplio 500" (TUS-A500; Toshiba Medical Systems Corporation), with usage of $3.5 \mathrm{MHz}$ phase probe at discharge and at 6-month observation period. Left ventricular (LV) end diastolic volume (LVEDV), LV end systolic volume (LV ESV), and LV ejection fraction (LVEF) measurements were taken according to Simpson's method per contemporary recommendation. The left atrial diameter (LAD) and left atrial volume (LAV) were determined according to contemporary protocol ${ }^{26}$. LV myocardial mass (LVMM) was calculated in an automatic manner per protocol of echocardiogram evaluation. LV global longitudinal strain $\left(e^{c}\right)$ and early transmitral velocity (E) were measured by tissue Doppler imaging technique and impulse transmitral Doppler regime at baseline and at 6 months per the protocol.

\section{Determination of STEMI prognosis}

We used the TIMI score to validate the prognostic capacity after STEMI ${ }^{27}$. 
$1 \mathrm{~A}$
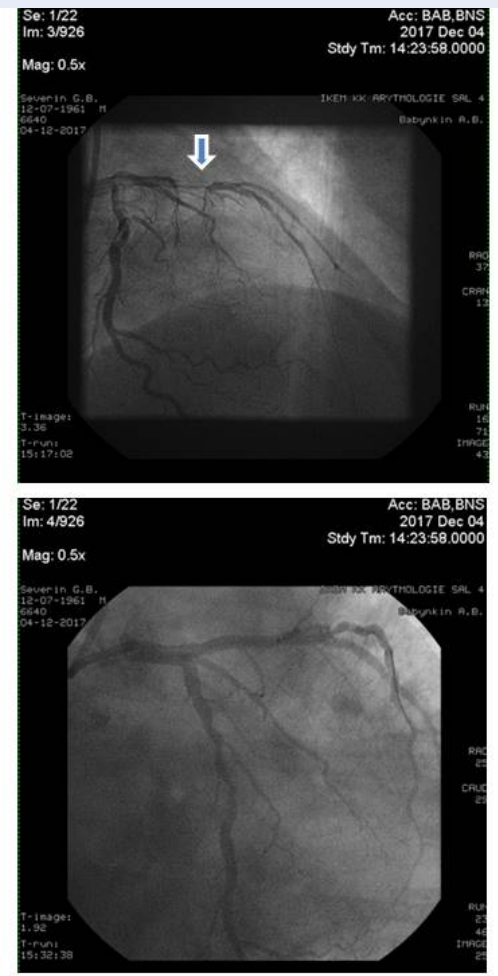

$1 B$
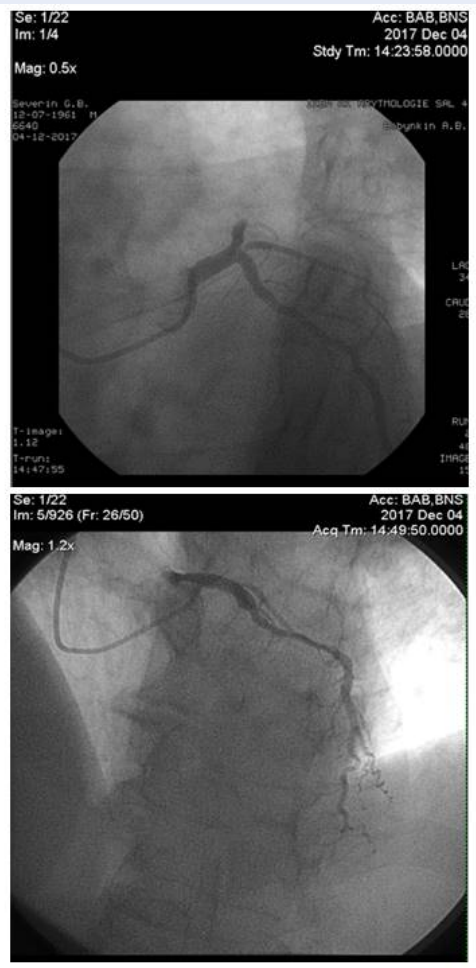

Figure 2: Complete revascularization in STEMI patients. Occlusion (arrow) and TIMI III blood flow restoring through culprit artery with $\mathrm{PCI}$ (1A and 1B, respectively) and reperfusion through ischemia-relating stenosis (1C and 1D).

\section{SYNTAX score determination}

SYNTAX score (SS) was used to assess the severity of coronary atherosclerotic lesions; it was calculated by experienced interventional cardiologists accordingly $^{28}$.

\section{Determination of endpoint}

The endpoint was determined as combined events including CV death, recurrent angina, and newly diagnosed heart failure for 6 months after PCI. CV death was ascertained by personal or phone contact from the family doctor or the hospital where the patient died. The diagnosis of recurrent angina required the presence of clinical signs/symptoms or electrocardiographic changes. Hospitalization was ascertained by direct contact or phone call to the hospital where the patient was admitted. A discharge report or autopsy report was obligatorily reviewed before the endpoint determination.

\section{Blood samples}

Blood samples were drawn immediately before PCI and at 6 months following acute STEMI. Blood samples were centrifuged, serum was isolated within
30 min of sample acquisition, and the sera then frozen at $-70^{0} \mathrm{C}$ and stored in plastic tubes until they were shipped to the Laboratory of Immune-Chemical and Molecular Genetic Research of "L.T.Malaya TNI NAMSU" (Kharkiv, Ukraine).

The N-terminal brain natriuretic peptide (NTproBNP) levels were measured using a commercial kit for ELISA manufactured by Vector-Best (Russia Federation).

The levels of troponin I (Tn I) and creatine kinaseisoenzyme-MB (CK-MB) were measured with chemiluminescent immunoassay on Humalyzer 2000 (Mannheim, Germany). The range of TnI and CK-MB levels were $0.5-50 \mathrm{ng} / \mathrm{mL}$ and $0-500$ $\mathrm{mmol} / \mathrm{L}$, respectively. The intra-assay and inter-assay coefficients of variation were $<5 \%$.

The levels of VEGF-A were measured using a commercial kit for ELISA produced by (IBL International GMBH, Germany). The VEGF-A level ranged from 0 to $1000 \mathrm{pg} / \mathrm{mL}$. The intra-assay and inter-assay coefficients of variation were $<5 \%$.

High-sensitive C-reactive protein (hs-CRP) levels in serum were measured with a commercially available standard kit (R\&D Systems GmbH, Wiesbaden- 
Nordenstadt, Germany). The hs-CRP level range was 0-100 mg/L.

Total cholesterol (TC), low density lipoprotein (LDL) cholesterol, high density lipoprotein (HDL) cholesterol, and triglycerides (TG) were measured by direct enzymatic method (Roche P800 Analyzer, Basel, Switzerland). The intra-assay and inter-assay coefficients of variation were $<5 \%$.

\section{SNP G634G (rs 2010963) in VEGF-A gene de- termination}

DNA extraction and analysis was performed from peripheral blood leukocytes per the commercial kit protocol «CFX96 Touch» (BioRad Laboratories Pte.Ltd., Ciayp) and per the appropriate RT-PCR kit (Syntol, Russia Federation). Genotyping of the G634C VEGF-A gene polymorphism located in the promoter region was performed using primers and probes in real-time (RT) polymerase chain reaction (PCR). The primers used for VEGF-A (rs 2010963) polymorphism assay were: GAGAGAAGT CGAGGAAGAGAGAGA-3' (forward primer), CCCAAAAGCAGGTCA CTCACTT-3' (reverse primer), VEGF-A-FAM-5' - CCTGTCCCTTTCGC$3^{\prime}$, and VEGF-A-VIC-5' - CCTGTCGCTTTCGC-3'.

\section{Statistical analysis}

Statistical analyses were performed using SPSS for Windows v.23 (USA). Continuous variables were presented as mean \pm standard deviation (SD) when normally distributed, or median and interquartile range (IQR) if otherwise. The categorical variables were presented as frequencies ( $\mathrm{n}$ ) and percentages (\%). MannWhitney and Wald-Wolfowitz criteria were used for intergroup differences and quantitative values. The qualitative variables are expressed as percentages, and were compared with the $\chi 2$ test and exact F Fisher test. Allele frequencies were estimated, and all polymorphisms were tested for Hardy-Weinberg equilibrium. Correlations between G634G VEGF-A gene polymorphism, angiographic characteristics, hemodynamic performances, and biomarkers were assessed using rank-order correlation Spearman's test. We performed univariate and multiple variate log-regression analysis to determine variables that predict endpoint and cardiac remodeling. Beta coefficient, standard errors (SE), odds ratio (OR), and 95\% confidence interval (CI) for each factor were estimated. Factors for which P-values were calculated as $>0.5$ were not included in the multiple variate log-regression analysis. Survival analysis for clinical outcomes was performed using Kaplan-Meier curves and the log-rank test. All differences were considered statistically significant with 2 -tailed $<0.05$.

\section{RESULTS}

The observed frequencies of variants of G634C VEGF-A (rs 2010963) genotype among the entire STEMI patient population ( $\mathrm{n}=135)$ were $\mathrm{GG}=51.9 \%$ $(\mathrm{n}=70), \mathrm{GC}=47.4 \%(\mathrm{n}=64)$ and $\mathrm{CC}=0.7 \%(\mathrm{n}=1)$, respectively. There was a deviation from the HardyWeinberg equilibrium due to an excess of heterozygosity $(\chi 2=10.9, \mathrm{P}=0.00099)$. Healthy volunteers ( $\mathrm{n}=30$ ) had GG VEGF-A (rs 2010963) genotype in $53 \%(\mathrm{n}=16)$, CT VEGF-A (rs 2010963) genotype in $43 \%(\mathrm{n}=13)$, and CC VEGF-A (rs 2010963) genotype in $4 \%(\mathrm{n}=1)$ of the patient population, without significant deviation from expected frequencies $(\chi 2$ $=0.726, \mathrm{P}=0.394)$. The circulating levels of VEGF-A in healthy volunteers and STEMI patients at baseline, based on G634C VEGF-A (rs 2010963) genotypes, are reported in Figure 3.

General baseline clinical and procedural characteristics of the patient study population are reported in Table 1. There were no significant differences between both STEMI patient cohorts with respect to age, sex, or CV risk factors (hypertension, dyslipidemia, abdominal obesity, type 2 diabetes mellitus, smoking, and premature CV events in family anamnesis). Therefore, we did not find sufficient differences between frequencies in atrial fibrillation, stable and unstable angina prior to STEMI, II-IV Killip classes of heart failure, or GRACE and TIMI score points in both patient cohorts. Additionally, baseline medications were similar in both STEMI patient cohorts. However, STEMI patients with GG variant of VEGF-A gene rarely presented anterior localization of myocardial infarction (MI) and frequently exhibited posterior localization of MI than individuals with GC/CC VEGF-A genotypes, while there was not difference in right main coronary artery injury between the cohorts (Table 2). On the contrary, STEMI patients with GC/CC VEGF-A genotypes had frequent left main coronary artery injury.

Table 3 shows the reported baseline cardio dynamic characteristics and biomarkers in the patient study population. STEMI patients with GC/CC VEGFA genotypes had significantly increased LVEDV, LVESV, and $\mathrm{E} / \mathrm{e}^{c}$ ratio at baseline in comparison with patients who had GG VEGF-A genotype. We did not observe differences between the patient cohorts in terms of GFR, serum creatinine, peak TnI, CK-MB, NT-proBNP, lipid profile, or hs-CRP. However, circulating levels of VEGF-A were profoundly lower in STEMI patients who had the GC/CC VEGF-A genotypes.

Six-month observation showed that cardiac hemodynamic performances and levels of cardiac biomarkers 


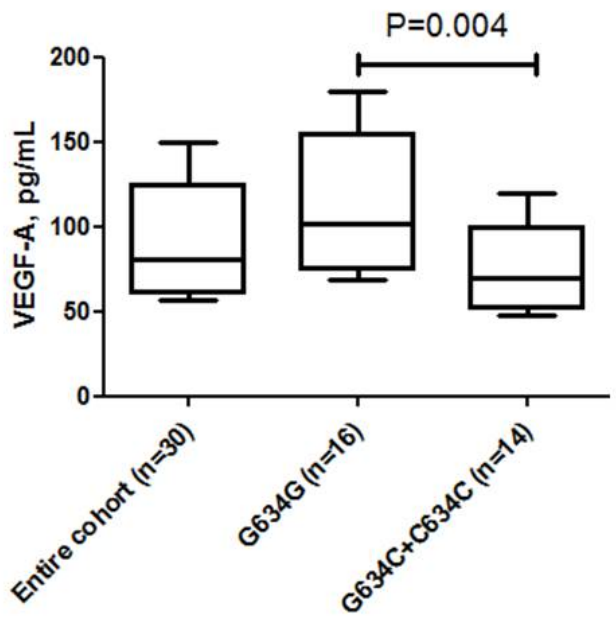

Healthy volunteers

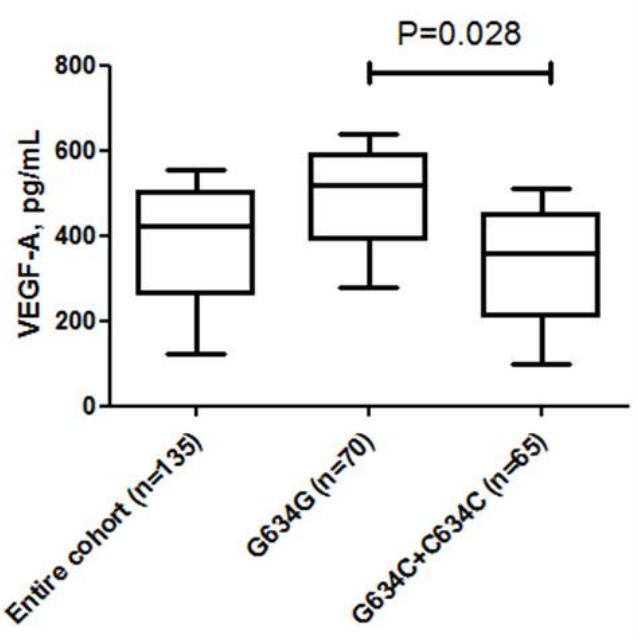

STEMI patients at baseline

Figure 3: The circulating levels of VEGF-A in healthy volunteers and STEMI patients depending on G634C VEGF-A (rs 2010963) genotypes.

(hs-CRP, VEGF-A, NT-proBNP) were similar in both patient cohorts, while $\mathrm{E} / \mathrm{e}^{\mathrm{c}}$ ratio was significantly increased in patients with GC/CC VEGF-A genotypes (Table 4). Dynamic changes of VEGF-A and NTproBNP levels in STEMI patients with variants of G634C VEGF-A polymorphism for 6 months are reported in Figure 4. Patients with GC/CC VEGF-A genotypes demonstrated both lowered 6-month levels of VEGF-A and increased 6-month levels of NTproBNP than individuals with GG VEGF-A genotype. Therefore, we found that within the 6-month period, 29 cases of pre-specified clinical outcomes occurred ( 9 cases and 20 cases in GG VEGF-A genotype and GC/CC VEGF-A genotype cohorts, respectively). Interestingly, significant differences between the cohorts were observed in terms of frequencies of MI, but not in other aspects of clinical outcomes.

\section{Correlations between G634G VEGF-A gene polymorphism, angiographic character- istics, hemodynamic performances, and biomarkers}

There were positive correlations between GC/CC VEGF-A genotype and combined endpoint $(r=0.58$;
$\mathrm{P}=0.0001)$, dynamic-increased NT-proBNP level for 6 months $(\mathrm{r}=0.42 ; \mathrm{P}=0.001)$, SYNTAX score $(\mathrm{r}=$ $0.34 ; \mathrm{P}=0.001)$, anterior STEMI $(\mathrm{r}=0.36 ; \mathrm{P}=0.003)$, LDL cholesterol $(\mathrm{r}=0.22 ; \mathrm{P}=0.032)$, TIMI score $(\mathrm{r}=$ $0.26 ; \mathrm{P}=0.012)$, atrial fibrillation $(\mathrm{r}=0.28 ; \mathrm{P}=0.001)$, unstable angina prior to STEMI $(\mathrm{r}=0.25 ; \mathrm{P}=0.047)$, $\mathrm{E} / \mathrm{e}^{\mathrm{c}}$ ratio $(\mathrm{r}=0.23 ; \mathrm{P}=0.048)$, and multiple coronary vessel injury $(r=0.26 ; P=0.002)$. There was inverted correlation between GC/CC VEGF-A genotype and dynamic-increased VEGF-A level for 6 months $(r=-$ $0.42 ; \mathrm{P}=0.001)$, and LV ejection fraction $(\mathrm{r}=-0.33 ; \mathrm{P}$ $=0.001)$ in acute STEMI patients.

Therefore, we found a correlation between LV ejection fraction and dynamic-increased NT-proBNP ( $\mathrm{r}$ $=-0.48 ; \mathrm{P}=0.003)$, and VEGF-A level for 6 months $(\mathrm{r}=0.46 ; \mathrm{P}=0.003)$ in STEMI patients with $\mathrm{GC} / \mathrm{CC}$ VEGF-A genotypes. However, it was not confirmed in patients with the GG variant of the VEGF-A genotype. Yet, multiple coronary artery injury was found to correlate with LV ejection fraction at baseline ( $\mathrm{r}=$ $0.33 ; \mathrm{P}=0.001)$, NT-proBNP $(\mathrm{r}=0.32 ; \mathrm{P}=0.001)$, dyslipidemia $(r=0.30 ; \mathrm{P}=0.002)$, type 2 diabetes mellitus $(r=0.28 ; \mathrm{P}=0.001)$, hs-CRP $(\mathrm{r}=0.26 ; \mathrm{P}=0.001)$, $\mathrm{E} / \mathrm{e}^{\mathrm{c}}$ ratio at baseline $(\mathrm{r}=0.24 ; \mathrm{P}=0.002)$, abdominal obesity $(r=0.23 ; \mathrm{P}=0.024)$, smoking $(\mathrm{r}=0.22$; 


\begin{tabular}{|c|c|c|c|c|}
\hline Variables & $\begin{array}{c}\text { Entire } \\
\text { population } \\
(\mathbf{n}=135)\end{array}$ & $\begin{array}{l}\text { Patients with } \\
\text { GG genotype } \\
\quad(n=70)\end{array}$ & $\begin{array}{c}\text { Patients with GC } \\
\text { and CC } \\
\text { genotypes } \\
(n=65)\end{array}$ & $\begin{array}{l}\chi^{2} \\
\text { P value }\end{array}$ \\
\hline Age, years & $59.2 \pm 8.92$ & $59.30 \pm 8.50$ & $59.69 \pm 8.85$ & $\chi^{2}=0.03 ; p=0.639$ \\
\hline Sex, male/female, n (\%) & $\begin{array}{l}109 / 26 \\
(80.7 / 19.3)\end{array}$ & $\begin{array}{l}57 / 13 \\
(81.4 / 19.6)\end{array}$ & $\begin{array}{l}52 / 13 \\
(80.0 / 20.0)\end{array}$ & $\chi^{2}=0.04 p=0,833$ \\
\hline Hypertension, n (\%) & $110(81.5)$ & $56(80.0)$ & $54(83.1)$ & $\chi^{2}=0.21 \mathrm{p}=0.646$ \\
\hline Dyslipidemia, n (\%) & $35(25.9)$ & $19(27.1)$ & $18(27.7)$ & $\chi^{2}=0.22 \mathrm{p}=0.688$ \\
\hline Abdominal obesity, n (\%) & $28(20.7)$ & $13(18.5)$ & $15(23.0)$ & $\chi^{2}=0.82 p=0.218$ \\
\hline Type 2 diabetes mellitus, $\mathrm{n}(\%)$ & $33(24.4)$ & $15(21.4)$ & $18(27.7)$ & $\chi^{2}=0.72 p=0.398$ \\
\hline Smoking, n (\%) & $65(48.1)$ & $33(47.1)$ & $32(49.2)$ & $\chi^{2}=0.06 \mathrm{p}=0.808$ \\
\hline $\begin{array}{l}\text { Premature } \mathrm{CV} \text { events in family } \\
\text { anamnesis, } \mathrm{n}(\%)\end{array}$ & $72(53.3)$ & $38(54.3)$ & $36(55.4)$ & $\chi^{2}=0.02 p=0.898$ \\
\hline $\begin{array}{l}\text { Stable angina prior to STEMI, } \mathrm{n} \\
(\%)\end{array}$ & $49(36.3)$ & $20(28.6)$ & $29(44.6)$ & $\chi^{2}=3.75 p=0.053$ \\
\hline $\begin{array}{l}\text { Unstable angina prior to STEMI, } \mathrm{n} \\
(\%)\end{array}$ & $46(34.1)$ & $19(27.1)$ & $27(41.5)$ & $\chi^{2}=3.11 \mathrm{p}=0.078$ \\
\hline Atrial fibrillation, n (\%) & $10(7.4)$ & $4(5.7)$ & $6(9.4)$ & $\chi^{2}=0.59 ; \mathrm{p}=0.326$ \\
\hline II-III Killip classes of HF, n (\%), & $26(19.3)$ & $12(17.1)$ & $14(21.5)$ & $\chi^{2}=0.42 p=0.518$ \\
\hline IV Killipclass of HF, n (\%) & $11(8.1)$ & $7(10.0)$ & $4(6.2)$ & $\chi^{2}=0,67 p=0.414$ \\
\hline \multicolumn{5}{|l|}{ Risk scores } \\
\hline GRACE Score, points & $150(120-172)$ & $143(117-170)$ & $152(119-176)$ & $\mathrm{P}=0.294$ \\
\hline TIMI score, points & $6(4-7)$ & $6(4-7)$ & $7(5-8)$ & $\mathrm{P}=0.66$ \\
\hline \multicolumn{5}{|l|}{ Baseline medications } \\
\hline Beta-blocker, n (\%) & $125(92.6)$ & $66(94.3)$ & $59(90.8)$ & $\chi^{2}=0.22 ; \mathrm{p}=0.762$ \\
\hline ACEI, n (\%) & $61(45.1)$ & $29(41.4)$ & $32(49.2)$ & $\chi^{2}=2.25 \mathrm{p}=0.068$ \\
\hline Ticagrelor, n (\%) & $135(100)$ & $70(100)$ & $65(100)$ & $\chi^{2}=0.016 ; \mathrm{p}=0.92$ \\
\hline Statins, n (\%) & $135(100)$ & $70(100)$ & $65(100)$ & $\chi^{2}=0.016 ; p=0.92$ \\
\hline
\end{tabular}

Notes: data are reported as $\mathrm{n}(\%)$, mean \pm standard deviation, and median (interquartile range). Mann-Whitney and Wald-Wolfowitz criteria were used for intergroup differences and quantitative values, respectively.

Abbreviations: ACEI: angiotensin-II converting enzyme inhibitor; HF: heart failure.

$\mathrm{P}=0.046)$, and male sex $(\mathrm{r}=0.22 ; \mathrm{P}=0.048)$. There were no observed significant associations between the GG variant of the VEGF-A genotype and prevalence of traditional $\mathrm{CV}$ risk factors.

\section{Determination of predictors for 6-month clinical endpoint}

The univariate linear regression (stepwise) analysis allowed for verifying TnI eak at admission, TIMI score, abdominal obesity, Killip HF class > II at admission, anterior localization of STEMI, atrial fibrilla- tion, GC/CC variants of VREGF-A gene, NT-proBNP at baseline, and dynamic changes in levels of NTproBNP and VEGF-A in serial measures as predictors for combined clinical endpoint (Table 5). Other variables did not embed into multivariate regressive analysis due to $\mathrm{P}>0.1$.

Unadjusted multivariate regressive logistic analysis showed peak TnI at admission, Killip class of $\mathrm{HF}$ $>2$, GC/CC polymorphisms in VEGF-A gene, and dynamic-increased NT-proBNP and VEGF-A levels for 6 months, which remained independent predic- 
Table 2: STEMI localization and angiographic characteristics

\begin{tabular}{|c|c|c|c|c|}
\hline Variables & $\begin{array}{r}\text { Entire } \\
\text { population } \\
(n=135)\end{array}$ & $\begin{array}{l}\text { Patients with } \\
\text { GG genotype } \\
\quad(\mathbf{n}=70)\end{array}$ & $\begin{array}{c}\text { Patients with GC } \\
\text { and CC } \\
\text { genotypes } \\
(n=65)\end{array}$ & $\begin{array}{l}\chi^{2} \\
\text { P value }\end{array}$ \\
\hline \multicolumn{5}{|l|}{ STEMI localization } \\
\hline Anterior wall, n (\%) & $59(43.7)$ & $24(34.3)$ & $35(53.8)$ & $\chi^{2}=5.24 \mathrm{p}=0.022$ \\
\hline Posterior wall, n (\%) & $53(39.3)$ & $34(48.6)$ & $19(29.2)$ & $\chi^{2}=5.29 \mathrm{p}=0.022$ \\
\hline Other, n (\%) & $23(17.0)$ & $12(17.1)$ & $11(16.9)$ & $\begin{array}{c}\chi^{2}=0.001 \mathrm{p}= \\
0.973\end{array}$ \\
\hline \multicolumn{5}{|l|}{ Infarct-related coronary artery } \\
\hline Left main coronary artery, $\mathrm{n}(\%)$ & $7(5.2)$ & $1(1.4)$ & $6(9.2)$ & $\chi^{2}=4.17 \mathrm{p}=0.041$ \\
\hline $\begin{array}{l}\text { Right main coronary artery, } \mathrm{n} \\
(\%)\end{array}$ & $27(20.0)$ & $10(14,3)$ & $17(26,2)$ & $\chi^{2}=2.97 \mathrm{p}=0.085$ \\
\hline $\begin{array}{l}\text { Circumflex coronary artery, } \mathrm{n} \\
\text { (\%) }\end{array}$ & $17(12.6)$ & $9(12.9)$ & $8(12.3)$ & $\chi^{2}=0.01 \mathrm{p}=0,923$ \\
\hline $\begin{array}{l}\text { Anterior interventricular artery, } \\
\mathrm{n}(\%)\end{array}$ & $31(23.0)$ & $12(17.1)$ & $19(29.2)$ & $\chi^{2}=2.78 p=0.095$ \\
\hline
\end{tabular}

Notes: data are reported as number and percentage.

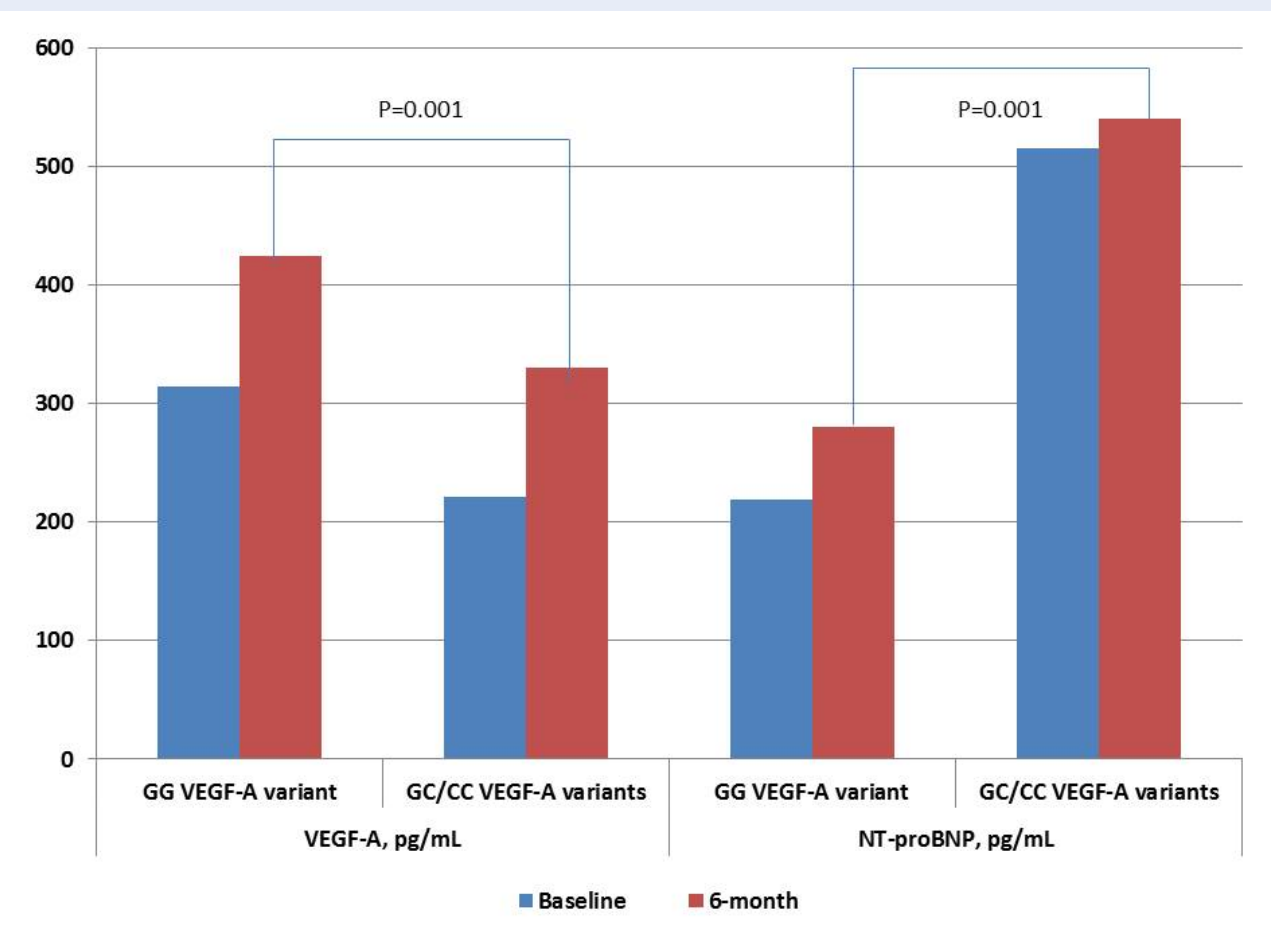

Figure 4: Dynamic changes of VEGF-A and NT-proBNP levels in STEMI patients with variants of G634C VEGFA polymorphism for 6 month. 
Table 3: Baseline cardio dynamic characteristics and biomarkers in patient study population

\begin{tabular}{|c|c|c|c|c|}
\hline Variables & $\begin{array}{l}\text { Entire population } \\
\qquad(\mathrm{n}=135)\end{array}$ & $\begin{array}{l}\text { Patients with GG } \\
\text { genotype } \\
\quad(n=70)\end{array}$ & $\begin{array}{l}\text { Patients with GC and } \\
\text { CC genotypes } \\
\quad(n=65)\end{array}$ & $\begin{array}{l}P \\
\text { value }\end{array}$ \\
\hline \multicolumn{5}{|l|}{ Hemodynamics } \\
\hline SBP, mm Hg & $135 \pm 22$ & $135 \pm 24$ & $136 \pm 27$ & 0.428 \\
\hline DBP, mm Hg & $80 \pm 12$ & $79 \pm 13$ & $82 \pm 14$ & 0.154 \\
\hline LVEDV, mL & $147 \pm 25$ & $138 \pm 31$ & $151 \pm 33$ & 0.044 \\
\hline LVESV, mL & $68 \pm 24$ & $63 \pm 23$ & $73 \pm 31$ & 0.039 \\
\hline LVEF, \% & $50 \pm 10$ & $51 \pm 13$ & $49 \pm 10$ & 0.250 \\
\hline E/e', units & $12.50 \pm 1.17$ & $10.20 \pm 1.21$ & $13.90 \pm 1.60$ & 0.046 \\
\hline LVMMI, $\mathrm{g} / \mathrm{m}^{2}$ & $149.30 \pm 44.32$ & $148.78 \pm 46.82$ & $157.28 \pm 47.10$ & 0.607 \\
\hline \multicolumn{5}{|l|}{ Biomarkers } \\
\hline Creatinine, $\mu \mathrm{mol} / \mathrm{L}$ & $98.13[86.90-119.30]$ & $96.75[86.30-113.20]$ & $104.40[88.10-123.60]$ & 0.274 \\
\hline GFR, $\mathrm{ml} / \mathrm{min}$ & $69.67[58.40-87.63]$ & $71.00[61.00-89.00]$ & $67.50[56.00-88.00]$ & 0.445 \\
\hline hs-CRP, mg/L & $12.04 \pm 4.77$ & $11.82 \pm 5.19$ & $12.53 \pm 5.03$ & 0.531 \\
\hline VEGF-A, pg/mL & $420.81[123.76-553.19]$ & 314.01 [159.94 - 627.66] & $221.28[77.58-440.82]$ & 0.045 \\
\hline Peak TnI, ng/ml & $18.4[5.44-77.3]$ & $17.70[6.77-99.00]$ & $23.07[4.07-75.50]$ & 0.914 \\
\hline CK-MB, mmol/L & $106.80[51.20-290.40]$ & $121.05[42.30-275.05]$ & $87.00[44.90$ - 300.10] & 0.458 \\
\hline $\begin{array}{l}\text { NT-proBNP, } \\
\text { pmol/L }\end{array}$ & $480.26[116.81-1558.31]$ & $219.34[75.70-440.82]$ & $515.56[109.91-1727.77]$ & 0.821 \\
\hline \multicolumn{5}{|l|}{$\begin{array}{l}\text { Lipid } \\
\text { profile }\end{array}$} \\
\hline $\mathrm{TC}, \mathrm{mmol} / \mathrm{l}$ & $4.95[3.97-5.71]$ & $4.97[3.98-5.64]$ & $4.91[4.10-5.63]$ & 0.85 \\
\hline $\mathrm{HDL}, \mathrm{mmol} / \mathrm{l}$ & $1.09[0.90-1.28]$ & $1.12[0.90-1.31]$ & $1.14[0.90-1.20]$ & 0.761 \\
\hline LDL, mmol/l & $3.00[2.11-3.71]$ & $2.97[2.11-3.67]$ & $3.01[2.07-3.99]$ & 0.303 \\
\hline $\mathrm{TG}, \mathrm{mmol} / \mathrm{l}$ & $1.53[1.17-2.02]$ & $1,90[1.19-2.06]$ & $1.61[1.13-1.91]$ & 0.023 \\
\hline
\end{tabular}

Notes: data are reported as $\mathrm{n}(\%)$, mean \pm standard deviation, and median [interquartile range]. Mann-Whitney and Wald-Wolfowitz criteria were used for intergroup differences.

Abbreviations: SBP: systolic blood pressure; DBP: diastolic blood pressure; LVEDV: left ventricular end diastolic volume; LVESV: left ventricular end systolic volume; LVEF: left ventricular ejection fraction; LVMM: left ventricular myocardial mass; LDL: low-density lipoprotein; HDL: high-density lipoprotein; TG: triglycerides; Tn: cardiac troponin; hs-CRP: high sensitive C-reactive protein; GRF: glomerular filtration rate; VEGF-A: vascular endothelial growth factor; NT-proBNP: N-terminal brain natriuretic peptide; CK-MB: creatinkinase isoform MB.

tors for the combined endpoint (Table 5). After adjustment for dynamic changes of NT-proBNP and VEGF-A levels for 6 months, we found that GC/CC polymorphisms of the VEGF-A gene was an independent predictor of poor clinical outcome $(\beta$-coefficient $=1.6635$; odds ratio $=2.8244 ; 95 \%$ confidence interval $=1.2649-11.2972 ; \mathrm{P}=0.0001)$. 
Table 4: 6-month cardio dynamic characteristics, levels of biomarkers and clinical outcomes in patient study population

\begin{tabular}{|c|c|c|c|c|}
\hline Variables & $\begin{array}{l}\text { Entire population } \\
\qquad(\mathrm{n}=135)\end{array}$ & $\begin{array}{l}\text { Patients with GG } \\
\text { genotype } \\
(\mathbf{n}=70)\end{array}$ & $\begin{array}{c}\text { Patients with GC and CC } \\
\text { genotypes } \\
(n=65)\end{array}$ & $P$ value \\
\hline \multicolumn{5}{|l|}{ Hemodynamics } \\
\hline SBP, mm Hg & $132 \pm 15$ & $136 \pm 22$ & $130 \pm 14$ & 0.186 \\
\hline DBP, mm Hg & $82 \pm 12$ & $82 \pm 14$ & $82 \pm 11$ & 0.484 \\
\hline LVEDV, mL & $155 \pm 20$ & $145 \pm 39$ & $158 \pm 31$ & 0.261 \\
\hline LVESV, mL & $78 \pm 24$ & $70 \pm 25$ & $81 \pm 39$ & 0.308 \\
\hline LVEF, \% & $50 \pm 8$ & $52 \pm 8$ & $49 \pm 12$ & 0.220 \\
\hline E/e', units & $13.30 \pm 1.17$ & $10.90 \pm 1.18$ & $15.70 \pm 1.25$ & 0.038 \\
\hline \multicolumn{5}{|l|}{ Biomarkers } \\
\hline hs-CRP, mg/L & $9.82 \pm 4.00$ & $8.54+3.20$ & $10.43 \pm 4.10$ & 0.662 \\
\hline VEGF-A, pg/mL & $\begin{array}{c}406.70 \\
{[210.50-523.71]}\end{array}$ & $\begin{array}{c}424.56 \\
{[230.60-556.93]}\end{array}$ & $\begin{array}{c}330.24 \\
{[162.80-472.14]}\end{array}$ & 0.129 \\
\hline NT-proBNP, pg/mL & $\begin{array}{c}388.29 \\
{[151.70-920.50]}\end{array}$ & $\begin{array}{c}280.29 \\
{[81.39-718.34]}\end{array}$ & $\begin{array}{c}540.01 \\
{[461.99-1217.31]}\end{array}$ & 0.074 \\
\hline \multicolumn{5}{|l|}{ Clinical outcomes } \\
\hline $\begin{array}{l}\text { Admission due to } \\
\text { HF, n (\%) }\end{array}$ & $6(4.4)$ & $1(1.4)$ & $5(7.7)$ & 0.088 \\
\hline MI, n (\%) & $14(10.4)$ & $5(7.1)$ & $9(13.8)$ & 0.048 \\
\hline Death, n (\%) & $9(6.7)$ & $3(4.3)$ & $6(9.2)$ & 0.160 \\
\hline Total, n (\%) & $29(21.4)$ & $9(12.9)$ & $20(30.8)$ & 0.020 \\
\hline
\end{tabular}

Notes: data are reported as $\mathrm{n}(\%)$, mean \pm standard deviation, and median [interquartile range]. Mann-Whitney and Wald-Wolfowitz criteria were used for comparisons

Abbreviations: SBP: systolic blood pressure; DBP: diastolic blood pressure; LVEDV: left ventricular end diastolic volume; LVESV: left ventricular end systolic volume; LVEF: left ventricular ejection fraction; hs-CRP: high sensitive C-reactive protein; GRF: glomerular filtration rate; VEGF-A: vascular endothelial growth factor; NT-proBNP: N-terminal brain natriuretic peptide; HF: heart failure. 
Table 5: The factors contributing 6-month combined end point after STEMI: The results of univariate and multivariate linear regressions

\begin{tabular}{|c|c|c|c|c|c|c|c|c|}
\hline \multirow[t]{4}{*}{ Variable } & \multicolumn{8}{|c|}{ Depending variable: combined clinical end point } \\
\hline & \multirow{2}{*}{\multicolumn{4}{|c|}{$\begin{array}{l}\text { Univariate linear regressive analysis } \\
\qquad\left(\chi^{2}=61.293 ; P<0.0001\right)\end{array}$}} & \multirow{2}{*}{\multicolumn{4}{|c|}{$\begin{array}{l}\text { Multivariate linear regressive analysis } \\
\qquad\left(\chi^{2}=32.140 ; \mathrm{P}<0.0001\right)\end{array}$}} \\
\hline & & & & & & & & \\
\hline & $\beta$-coefficient & OR & $95 \% \mathrm{C}$ & $\mathrm{P}$ & $\beta$-coefficient & OR & $95 \% \mathrm{C}$ & $\mathrm{P}$ \\
\hline Peak Tn at admission & -1.8578 & 1.0111 & $1.0007-1.0215$ & 0.0358 & -0.012610 & 1.0127 & $1.0016-1.0239$ & 0.0247 \\
\hline Peak CK-MB at admission & 0.47640 & 1.0254 & $1.0180-1.104$ & 0.4820 & & & 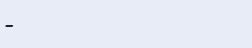 & \\
\hline SYNTAX score & 0.98677 & 1.0028 & $1.0002-1.006$ & 0.6884 & & & - & \\
\hline TIMI score & 1.37250 & 1.8970 & $0.9720-2.880$ & 0.0410 & 1.17280 & 1.0940 & $1.010-1.3240$ & 0.0520 \\
\hline Killip class of HF > II & 0.9874 & 1.3725 & $0.0536-2.5869$ & 0.3179 & 2.23331 & 9.3307 & $2.4408-35.6689$ & 0.0011 \\
\hline Smoking & -0.49898 & 0.6071 & $0.0328-11.2510$ & 0.7376 & & & - & \\
\hline Dyslipidemia & 0.4582 & 0.8848 & $0.6638-1.1255$ & 0.6388 & & & - & \\
\hline $\mathrm{T} 2 \mathrm{DM}$ & 2.98372 & 19.7611 & $0.8341-46.1875$ & 0.0647 & & & - & \\
\hline Abdominal obesity & 1.12320 & 2.1448 & $0.4607-3.8995$ & 0.0383 & 1.02 & 1.9560 & $0.0774-3.4539$ & 0.0526 \\
\hline Stable angina prior to STEMI & 0.43968 & 1.5522 & $0.3988-6.0419$ & 0.5260 & & & 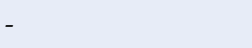 & \\
\hline Unstable angina prior to STEMI & 1.55459 & 0.2113 & $0.0149-2.9884$ & 0.2501 & & & - & \\
\hline Anterior STEMI & 1.98807 & 7.3014 & $1.1181-47.6782$ & 0.0378 & 1.85810 & 5.2064 & $0.0122-21.4412$ & 0.0586 \\
\hline Atrial fibrillation & 1.00347 & 0.1349 & $0.0157-1.1565$ & 0.0476 & 1.00254 & 0.1104 & $0.0120-1.1433$ & 0.0662 \\
\hline Multiple coronary vessel injury & 1.37022 & 3.9362 & $0.8228-18.8312$ & 0.0862 & & & - & \\
\hline \multicolumn{9}{|l|}{ A gene } \\
\hline $\mathrm{E} / \mathrm{e}^{c}$ at baseline & 0.35360 & 0.9160 & $1.0136-1.1630$ & 0.0870 & & & - & \\
\hline NT-proBNP at baseline & 1.18440 & 1.7044 & $1.0633-2.954$ & 0.03420 & 1.17230 & 1.0144 & $1.0330-1.1422$ & 0.0620 \\
\hline $\begin{array}{l}\text { Dynamic increased NT-proBNP } \\
\text { level for } 6 \text { month }\end{array}$ & 1.21370 & 1.8692 & $1.1354-4.8264$ & 0.02427 & 1.09377 & 1.2177 & $1.0464-3.5569$ & 0.0357 \\
\hline $\begin{array}{l}\text { Dynamic increased VEGF-A level } \\
\text { for } 6 \text { month }\end{array}$ & -0.005467 & 1.0055 & $1.0007-1.0103$ & 0.0241 & -0.0015896 & 1.0016 & $1.0002-1.0029$ & 0.0201 \\
\hline
\end{tabular}


Kaplan-Meier analysis for endpoint accumulation trends in STEMI patients with variants of VEGF-A gene

Kaplan-Meier curves have demonstrated that STEMI patients with GG VEGF-A genotype had a lower frequency of clinical combined endpoint accumulation when compared to those who had GC/CC VEGF-A genotypes (Log-rank p = 0.02) (Figure 5).

\section{DISCUSSION}

The results of our study maintain the hypothesis that the GG variant of the VEGF-A gene is able to mediate myocardial protection via higher levels of VEGF$\mathrm{A}$ in peripheral blood, prior to STEMI as well as for 6 months after the event. Consequently, GC/CC variants of the VEGF-A gene, which were associated with lowered basic and post-event levels of VEGF-A, have demonstrated close relation to accumulation of combined clinical endpoint for 6 months. We first revealed that complete revascularization in STEMI patients having the GC/CC variants of the VEGFA gene can draw unexpectedly worse clinical results for follow-up than those patients with the GG variant of the VEGF-A gene. Interestingly, at baseline, both STEMI patient cohorts did not significantly differ from each other with regards to traditional CV risk factors, several biomarkers levels (including CRP and NT-proBNP), and severity of coronary atherosclerosis Meanwhile left main coronary artery injury was determined frequently in patients with GC/CC variants of VEGF-A gene.

Previous animal and clinical studies have shown that VEGF-A exhibited powerful cardiac reparative effect, protected from ischemia/reperfusion injury, and reduced myocardial edema and MI size. Indeed, the cardiac macrophages recruited by pro-inflammatory cytokines are certainly conductors of these protective impacts $^{29-31}$. Moreover, VEGF-A levels were independently associated with microvascular obstruction during STEMI ${ }^{31}$. However, our results may indirectly confirm evidence of VEGF-A acting as a protector of endovascular edema and microvascular obstruction, preventing adverse LV remodeling. Indeed, Ferraro B et al. (2019) ${ }^{29}$ reported that the VEGF-A levels were significantly and inversely correlated with LVEF at 6-month follow-up. Our results corroborate with this evidence, although we did not find strong correlation between the VEGF-A levels at baseline and LVEF at baseline. However, there were strong interrelations between GG VEGF-A genotype and circulating levels of VEGF-A. Probably, the role of variants of VEGF-A gene has become crucial for postevent periods and correspond to maintaining higher
VEGF-A levels in peripheral blood to prevent adverse LV remodeling through lowered risk of distal coronary obstruction $^{32}$. However, angiographic parameters and coronary anatomy in STEMI patients who were effectively treated with primary PCI (with TIMI-III restoring blood flow and complete reperfusion) did not strongly correlate to a risk of late microvascular obstruction, which remained an independent predictor of LV remodeling, mortality following STEMI, and all-cause mortality even after further adjustment for infarct size ${ }^{33,34}$. In this context, a prediction of follow-up survival during STEMI treated effectively with PCI seems to be a credible tool for risk stratification of STEMI patients, and GC/CC VEGF-A genotypes could be discussed as a prognosticator for poor clinical outcomes. However, there is no optimal methodology that best predicts the surrogate outcome marker of LV function and survival in post-STEMI patients ${ }^{35,36}$

The strength of our investigation is the enrollment in the study of STEMI patients with normal and preserved LVEF who were candidates for complete reperfusion therapy with PCI. There are limiting data which were able to predict CV events and late LV remodeling in post-STEMI individuals without clinical significance and declining of LV pump function and symptomatic $\mathrm{HF}^{36}$. The results of our study have revealed that presentation of GC/CC variants of VEGF-A gene predicted well the tendency for LFEF and LF diastolic filling to worsen. Note that these results may be interpreted in the context of late microvascular obstruction that frequently follows complete PCI. Indeed, our results showed that combined the clinical endpoint occurred predominantly due to new MI, but not any other reasons. Probably, GC/CC variants of VEGF-A gene were not able to support vascular protection with adequate VEGF-A production after STEMI and could not protect microvascular obstruction, which led to MI in the follow-up period ${ }^{37}$.

It has been revealed that STEMI patients who might have microvascular obstruction had higher peak TnT and lower LVEF because of an increased LVEDV ${ }^{38}$. Although an association between GC/CC variants of the VEGF-A gene exists, the risk of CAD and quality of life in the general population has been previously determined ${ }^{39,40}$ There were not close associations between severity of atherosclerosis, number of damaged coronary artery, and GC/CC VEGF-A genotype in the STEMI patient population ${ }^{41}$. On contrary, there are other findings (which are clarified positive associations) between the culprit artery lesion localization, MI size, and microvascular obstruction ${ }^{42,43}$. Because of there are close interrelationships between VEGF-A 


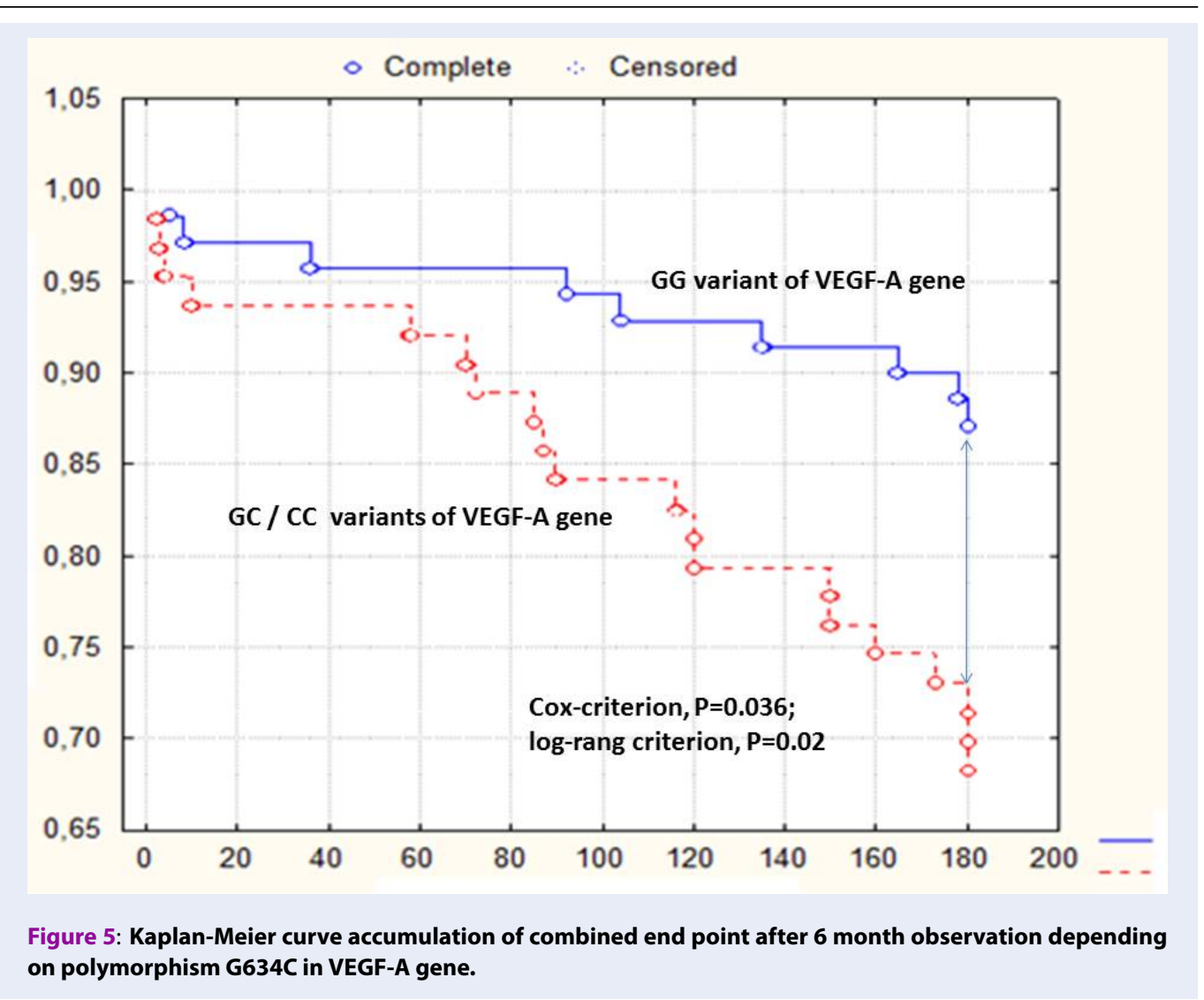

tissue expression and serum levels, microvessel density, ROS production, and expression of vascular endothelial cadherin in re-perfused myocardium ${ }^{44}$, the role of variants of VEGF-A genotypes in tissue protection in STEMI has been suggested.

In this context, it has remained largely unclear as to the exact molecular mechanisms which mediate the causative relation of VEGF-A to vascular and myocardium protection in post-STEMI patients. It has been postulated that elevated levels of VEGF$\mathrm{A}$ in post-STEMI patients is a result of myocardial ischemia/hypoxia induced by microvascular dysfunction after distal coronary artery embolization and vascular inflammation. In fact, early microvascular obstruction is accompanied with decline of LVEF, whereas late microvascular dysfunction in post-STEMI individuals can associate with LV diastolic dysfunction without reduced $\operatorname{LVEF}^{45,46}$. Large clinical studies are required to clearly understand the role of genetic polymorphisms of the VEGF-A gene in mediating endogenous reparation and tissue protection. However, we believe that determination of the GC/CC VEGF-A genotype could provide relevant prognostic insights, leading to improved short-term and long-term risk stratification in STEMI patients treated with complete revascularization.

There are several study limitations. First, the small sample size does not allow for the analysis to be conducted in detail in greater subgroups. Additionally, it does not allow for fully interpreting and understanding the causes that lead to the appearance of MACEs directly related to the new MI. Second, it would be optimal to use late gadolinium-enhanced magnetic resonance images to determine an interrelationship between microvascular obstruction and increased risk of adverse CV events in post-STEMI patients. Third, it has still not fully understand whether attenuated plaques and well-positioned stents were causes for adverse outcomes. Yet, we have measured total VEGF-A levels, but we did not determine fractions of VEGFA which were found to mediate different impacts on myocardium and vasculature. Finally, we believe that these study limitations will not be able to sufficiently diminish the value of the results of our investigation.

\section{CONCLUSION}

The G634C polymorphism of the VEGF-A gene was found to be an independent predictor for 6-month 
clinical combined endpoints in STEMI patients after successful primary PCI.

\section{LIST OF ABBREVIATIONS}

ADA: American Diabetic Association

CAD: coronary artery disease

CI: 95\% confidence interval

CK-MB: creatinine kinase isoenzyme-MB

CV: cardiovascular

DBP: diastolic blood pressure

E: early transmitral velocity

é: global longitudinal LV strain

ECS: European Cardiology Society

EDV: end diastolic volume

EF: ejection fraction

ESV: end systolic volume

HDL: high density lipoprotein

HF: heart failure

IQR: interquartile range

LDL: low density lipoprotein

LV: left ventricular

MACE: major cardiovascular events

NT-proBNP: NT-fragment pro-natriuretic peptide

PCI: percutaneous coronary intervention

ROS: reactive oxygen species

SBP: systolic blood pressure

SD: standard deviation

STEMI: ST segment elevation myocardial infarction T2DM: type 2 diabetes mellitus

TC: total cholesterol

TG: triglycerides

\section{ACKNOWLEDGMENTS}

There are no previous presentations of the information reported in the article.

\section{AUTHOR'S CONTRIBUTIONS}

Study Design: Inna M. Kutia, Mykola P. Kopytsya, Yaroslava V. Hilova, Alexander E. Berezin. Data Collection: Inna M. Kutia, Mykola P. Kopytsya, Yaroslava V. Hilova; Olga V Petyunina. Statistical Analysis: Inna M. Kutia, Alexander E. Berezin. Data Interpretation: Inna M. Kutia, Mykola P. Kopytsya, Yaroslava V. Hilova, Olga V Petyunina; Alexander E. Berezin. Manuscript Preparation: Inna M. Kutia, Mykola P. Kopytsya, Yaroslava V. Hilova, Olga V Petyunina; Alexander E. Berezin. Literature Search: Inna M. Kutia; Alexander E. Berezin. All authors read and approved the final manuscript.

\section{FUNDING}

The study is a fragment of the research project: “To study the biochemical, genetic mechanisms of reperfusion damage of the myocardium and to assess the cardioprotective effect of antiplatelet therapy in acute myocardial infarction", State Registration No. 0117U003028 / Ukraine

No commercial funds were collected for this study.

\section{AVAILABILITY OF DATA AND MATERIALS}

Data and materials used and/or analysed during the current study are available from the corresponding author on reasionable request.

\section{ETHICS APPROVAL AND CONSENT TO PARTICIPATE}

This study was conducted in accordance with the amended Declaration of Helsinki. The institutional review board approved the study (Protocol №6, $30.05 .2017)$, and all participants provided written informed consent.

\section{CONSENT FOR PUBLICATION}

Not applicable.

\section{COMPETING INTERESTS}

The authors declare that they have no competing interests.

\section{REFERENCES}

1. Thomas MP, Bates ER. Update on primary $\mathrm{PCI}$ for patients with STEMI. Trends Cardiovasc Med. 2017;27(2):95-102. PMID: 27450063. Available from: https://doi.org/10.1016/j.tcm.2016. 06.010.

2. Banga S, Gumm DC, Kizhakekuttu TJ, Kizhakekuttu TJ, Emani VK, Singh S, et al. Left Ventricular Ejection Fraction along with Zwolle Risk Score for Risk Stratification to Enhance Safe and Early Discharge in STEMI Patients Undergoing Primary Percutaneous Coronary Intervention: A Retrospective Observational Study. Cureus. 2019;11(7):e5272. Available from: https://doi.org/10.7759/cureus.5272.

3. Tarantini G, D'Amico G, Brener SJ, Tellaroli P, Basile M, Schiavo A, et al. Survival After Varying Revascularization Strategies in Patients With ST-Segment Elevation Myocardial Infarction and Multivessel Coronary Artery Disease: A Pairwise and Network Meta-Analysis. JACC Cardiovasc Interv. 2016;9(17):1765-1776. PMID: 27609250. Available from: https://doi.org/10.1016/j.jcin. 2016.06.012.

4. Song F, Wu Q, Chen XP. New debate of revascularization strategy of non-infarct-related artery lesions in patients with ST-segment elevation myocardial infarction and cardiogenic shock: decoding the CULPRIT-SHOCK trial, not enough to challenge the current guidelines. Chin Med J (Engl). 2019;132(5):613-615. PMID: 30807360. Available from: https: //doi.org/10.1097/CM9.0000000000000112.

5. Thiele H, Akin I, Sandri M, Fuernau G, Waha S, Meyer-Saraei R, et al. $\mathrm{PCl}$ strategies in patients with acute myocardial infarction and cardiogenic shock. N Engl J Med. 2017;377:24192432. PMID: 29083953. Available from: https://doi.org/10. 1056/NEJMoa1710261. 
6. Windecker $\mathrm{S}$, Kolh $\mathrm{P}$, Alfonso $\mathrm{F}$, Collet JP, Cremer J, et al. Authors/Task Force members. 2014 ESC/EACTS Guidelines on myocardial revascularization: the Task Force on Myocardial Revascularization of the European Society of Cardiology (ESC) and the European Association for Cardio-Thoracic Surgery (EACTS) developed with the special contribution of the European Association of Percutaneous Cardiovascular Interventions (EAPCI). Eur Heart J. 2014;35:2541-2619. PMID: 25173339. Available from: https://doi.org/10.1093/eurheartj/ ehu278.

7. Park DW, Clare RM, Schulte PJ, Pieper KS, Shaw LK, Califf $\mathrm{RM}$, et al. Extent, location, and clinical significance of noninfarct-related coronary artery disease among patients with ST-elevation myocardial infarction. JAMA. 2014;312:20192027. PMID: 25399277. Available from: https://doi.org/10. 1001/jama.2014.15095.

8. Kosmidou I, McAndrew T, Redfors B, Embacher M, Dizon JM, Mehran R, et al. Correlation of Admission Heart Rate With Angiographic and Clinical Outcomes in Patients With Right Coronary Artery ST-Segment Elevation Myocardial Infarction Undergoing Primary Percutaneous Coronary Intervention: HORIZONS-AMI (The Harmonizing Outcomes With Revascularization and Stents in Acute Myocardial Infarction) Trial. J Am Heart Assoc. 2017;6(7). PMID: 28724652. Available from: https://doi.org/10.1161/JAHA.117.006181.

9. Gajanana D, Weintraub WS, Kolm P, Rogers T, lantorno M, BenDor l, et al. Trends in Death Rate 2009 to 2018 Following Percutaneous Coronary Intervention Stratified by Acuteness of Presentation. Am J Cardiol. 2019;9149(19):30890-30892. PMID: 31547993. Available from: https://doi.org/10.1016/j.amjcard. 2019.07.059.

10. Hueso L, Rios-Navarro C, Ruiz-Sauri A, Chorro FJ, Nunez J, Sanz MJ, et al. Dynamics and implications of circulating antiangiogenic VEGF-A165b isoform in patients with ST-elevation myocardial infarction. Sci Rep. 2017;7(1):9962. PMID: 28855597. Available from: https://doi.org/10.1038/s41598017-10505-9.

11. Grąbczewska Z, Dębski R, Góralczyk K, Sukiennik A, Świątkiewicz I, Kubica J. Associations between selected angiographic parameters and the number of $\mathrm{CD}_{3} 4^{+}$ cells and plasma levels of vascular endothelial growth factor and angiogenin in patients with ST-segment elevation myocardial infarction. Pol Arch Med Wewn. 2015;125(3):132-140. PMID: 25643927. Available from: https://doi.org/10.20452/pamw.2703.

12. Kim BH, Ko YG, Her AY, Kim JS, Hwang KC, Shin DH, et al. Serial plasma levels of angiogenic factors in patients with ST-segment elevation myocardial infarction undergoing primary percutaneous coronary intervention. Korean Circ J. 2012;42(7):464-470. PMID: 22870080. Available from: https: //doi.org/10.4070/kcj.2012.42.7.464.

13. Olsson AK, Dimberg A, Kreuger J, Claesson-Welsh L. VEGF receptor signalling - in control of vascular function. Nat Rev Mol Cell Biol. 2006;7:359-371. PMID: 16633338. Available from: https://doi.org/10.1038/nrm1911.

14. Ferrara N, Gerber HP, LeCouter J. The biology of VEGF and its receptors. Nat Med. 2003;9:669-676. PMID: 12778165. Available from: https://doi.org/10.1038/nm0603-669.

15. Sato A, Yoshihisa A, Yokokawa T, Shimizu T, Nakamura $Y$, Misaka $\mathrm{T}$, et al. The association between circulating antiangiogenic isoform of vascular endothelial growth factor and clinical profiles in patients with peripheral artery disease. Int J Cardiol. 2016;207:368-369. PMID: 26820370. Available from: https://doi.org/10.1016/j.ijcard.2016.01.189.

16. Cochain C, Channon KM, Silvestre JS. Angiogenesis in the infarcted myocardium. Antioxid Redox Signal. 2013;18:11001113. PMID: 22870932. Available from: https://doi.org/10. 1089/ars.2012.4849.

17. Pyda M, Korybalska K, Ksiazek K, Grajek S, Lanocha M, Lesiak M, et al. Effect of heparin on blood vascular endothelial growth factor levels in patients with ST-elevation acute myocardial infarction undergoing primary percutaneous coronary inter- vention. Am J Cardiol. 2006;98(7):902-905. PMID: 16996870. Available from: https://doi.org/10.1016/j.amjcard.2006.04.028.

18. Liu KL, Lin SM, Chang CH, Chen YC, Chu PH. Plasma angiopoietin-1 level, left ventricular ejection fraction, and multivessel disease predict development of 1-year major adverse cardiovascular events in patients with acute ST elevation myocardial infarction - a pilot study. Int J Cardiol. 2015;182:155-160. PMID: 25577775. Available from: https: //doi.org/10.1016/j.ijcard.2014.12.172.

19. Al-Habboubi HH, Sater MS, Almawi AW, Al-Khateeb GM, Almawi WY. Contribution of VEGF polymorphisms to variation in VEGF serum levels in a healthy population. Eur Cytokine Netw. 2011;22:154-158. PMID: 21982816. Available from: https://doi.org/10.1684/ecn.2011.0289.

20. Watson CJ, Webb NJ, Bottomley MJ, Brenchley PE. Identification of polymorphisms within the vascular endothelial growth factor (VEGF) gene: correlation with variation in VEGF protein production. Cytokine. 2000;12(8):1232-1235. PMID 10930302. Available from: https://doi.org/10.1006/cyto.2000. 0692.

21. Ibanez B, James $S$, Agewall S, Antunes MJ, Bucciarelli-Ducci C, Bueno H, et al. ESC Scientific Document Group. 2017 ESC Guidelines for the management of acute myocardial infarction in patients presenting with ST-segment elevation: The Task Force for the management of acute myocardial infarction in patients presenting with ST-segment elevation of the European Society of Cardiology (ESC). Eur Heart J. 2018;39(2):119177. PMID: 28886621. Available from: https://doi.org/10.1093/ eurheartj/ehx393.

22. Kirby $A$, Gebski $V$, Keech $A C$. Determining the sample size in a clinical trial. Med J Aust. 2002. https://doiorg/105694/j132653772002tb04759x;177(5):256-257. PMID: 12197821.

23. Catapano AL, Graham I, Backer GD, Wiklund O, Chapman MJ, Drexel H, et al. ESC Scientific Document Group. 2016 ESC/EAS Guidelines for the Management of Dyslipidemias: The Task Force for the Management of Dyslipidemias of the European Society of Cardiology (ESC) and European Atherosclerosis Society (EAS) Developed with the special contribution of the European Association for Cardiovascular Prevention \& Rehabilitation (EACPR). Atherosclerosis. 2016;253:281-344. PMID: 27567407. Available from: https://doi.org/10.1093/eurheartj/ ehw272.

24. Williams B, Mancia G, Spiering W, Rosei EA, Azizi M, Burnier M, et al. ESC Scientific Document Group. 2018 ESC/ESH Guidelines for the management of arterial hypertension. Eur Heart J. 2018;39(33):3021-3104. PMID: 30165516. Available from: https://doi.org/10.1093/eurheartj/ehy339.

25. Ponikowski $\mathrm{P}$, Voors $\mathrm{AA}$, Anker SD, Bueno $\mathrm{H}, \mathrm{Cleland} J \mathrm{JF}$, Coats AJS, et al. ESC Scientific Document Group 2016 ESC guidelines for the diagnosis and treatment of acute and chronic heart failure: the task force for the diagnosis and treatment of acute and chronic heart failure of the European Society of Cardiology (ESC). Developed with the special contribution of the Heart Failure Association (HFA) of the ESC. Eur J Heart Fail. 2016;18:891-975. PMID: 27206819. Available from: https: //doi.org/10.1093/eurheartj/ehw128.

26. Jessup M, Drazner MH, Book W, Cleveland JCJ, Dauber I, Farkas S, et al. 2017 ACC/AHA/HFSA/ISHLT/ACP Advanced Training Statement on Advanced Heart Failure and Transplant Cardiology (Revision of the ACCF/AHA/ACP/HFSA/ISHLT 2010 Clinical Competence Statement on Management of Patients With Advanced Heart Failure and Cardiac Transplant): A Report of the ACC Competency Management Committee. J Card Fail. 2017;23(6):492-511. PMID: 28454730. Available from: https: //doi.org/10.1016/j.cardfail.2017.04.004.

27. Morrow DA, Antman EM, Charlesworth A, Cairns R, Murphy SA, de Lemos JA, et al. TIMI Risk Score for ST-Elevation Myocardial Infarction: A Convenient, Bedside, Clinical Score for Risk Assessment at Presentation. An Intravenous nPA for Treatment of Infarcting Myocardium Early II Trial Substudy. Circulation. 2000;102:2031-2037. PMID: 11044416. Available from: 
https://doi.org/10.1161/01.CIR.102.17.2031.

28. Kappetein AP, Dawkins KD, Mohr FW, Morice MC, Mack MJ, Russell ME, et al. Current percutaneous coronary intervention and coronary artery bypass grafting practices for three-vessel and left main coronary artery disease. Insights from the SYNTAX run-in phase. Eur J Cardiothorac Surg. 2006;29(4):486491. PMID: 16497510. Available from: https://doi.org/10.1016/ j.ejcts.2006.01.047.

29. Ferraro B, Leoni G, Hinkel R, Ormanns S, N NP, Ortega-Gomez $A$, et al. Pro-Angiogenic Macrophage Phenotype to Promote Myocardial Repair. J Am Coll Cardiol. 2019;73(23):2990-3002. PMID: 31196457. Available from: https://doi.org/10.1016/j. jacc.2019.03.503.

30. Dong J, Xu M, Zhang W, Che X. Effects of Sevoflurane Pretreatment on Myocardial Ischemia-Reperfusion Injury Through the Akt/Hypoxia-Inducible Factor 1-alpha (HIF-1 $\alpha$ )/Vascular Endothelial Growth Factor (VEGF) Signaling Pathway. Med Sci Monit. 2019;25:3100-3107. PMID: 31028241. Available from: https://doi.org/10.12659/MSM.914265.

31. Garcia R, Bouleti C, Sirol M, Logeart D, Monnot C, ArdidieRobouant C. VEGF-A plasma levels are associated with microvascular obstruction in patients with ST-segment elevation myocardial infarction. Int J Cardiol. 2019;291:19-24. PMID: 30910283. Available from: https://doi.org/10.1016/j.ijcard. 2019.02.067.

32. Berezin AE. Endogenous vascular repair system in cardiovascular disease: The role of endothelial progenitor cells . Australasian Medical J. 2019;12(2):42-48. Available from: https: //doi.org/10.21767/AMJ.2018.3464.

33. Wong DT, Leung MC, Richardson JD, Puri R, Bertaso AG, Williams $\mathrm{K}$, et al. Cardiac magnetic resonance derived late microvascular obstruction assessment post ST-segment elevation myocardial infarction is the best predictor of left ventricular function: a comparison of angiographic and cardiac magnetic resonance derived measurements. Int J Cardiovasc Imaging. 2012;28(8):1971-1981. PMID: 22310980. Available from: https://doi.org/10.1007/s10554-012-0021-9.

34. Waha S, Patel MR, Granger CB, Ohman EM, Maehara A, Eitel I, et al. Relationship between microvascular obstruction and adverse events following primary percutaneous coronary intervention for ST-segment elevation myocardial infarction: an individual patient data pooled analysis from seven randomized trials. Eur Heart J. 2017;38(47):3502-3510. PMID: 29020248. Available from: https://doi.org/10.1093/eurheartj/ehx414.

35. Berezin AE, Berezin AA. Dynamic Changes of Circulating Vascular Endothelial Growth Factor Levels in ST-Segment Elevation Myocardial Infarction: Controversies in Clinical Interpretation. Clin Trial Cardiol. 2019;6(1):1-4. Available from: https: //doi.org/10.15761/GMO.1000158.

36. Galea N, Dacquino GM, Ammendola RM, Coco S, Agati L, Luca LD, et al. Microvascular obstruction extent predicts major adverse cardiovascular events in patients with acute myocardial infarction and preserved ejection fraction. Eur Radiol. 2019;29(5):2369-2377. PMID: 30552479. Available from: https://doi.org/10.1007/s00330-018-5895-z.

37. Shiono $\mathrm{Y}$, Kubo T, Tanaka A, Tanimoto $\mathrm{T}$, Ota $\mathrm{S}$, Ino $\mathrm{Y}$, et al. Impact of attenuated plaque as detected by intravascular ul- trasound on the occurrence of microvascular obstruction af ter percutaneous coronary intervention in patients with STsegment elevation myocardial infarction. JACC Cardiovasc Interv. 2013;6(8):847-853. PMID: 23871509. Available from: https://doi.org/10.1016/j.jcin.2013.01.142.

38. Durante A, Laricchia A, Benedetti G, Esposito A, Margonato A, Rimoldi O, et al. Identification of High-Risk Patients After ST-Segment-Elevation Myocardial Infarction: Comparison Between Angiographic and Magnetic Resonance Parameters. Circ Cardiovasc Imaging. 2017;10(6). Available from: https: //doi.org/10.1161/CIRCIMAGING.116.005841.

39. Han X, Liu L, Niu J, Yan J, Zhang Z. Association between VEGF polymorphisms (936c/t, $-460 \mathrm{t} / \mathrm{c}$ and $-634 \mathrm{~g} / \mathrm{c}$ ) with haplotypes and coronary heart disease susceptibility. Int J Clin Exp Pathol. 2015;8(1):922-927.

40. Li L, Pan Y, Zhang D. Association of Genetic Polymorphisms on Vascular Endothelial Growth Factor and its Receptor Genes with Susceptibility to Coronary Heart Disease. Med Sci Monit. 2016;22:31-40. PMID: 26726843. Available from: https://doi. org/10.12659/MSM.895163.

41. Nia SK, Ziaee S, Boroumand MA, Anvari MS, Pourgholi L, Jalali A. The impact of vascular endothelial growth factor $+405 \mathrm{C} / \mathrm{G}$ polymorphism on long-term outcome and severity of coronary artery disease. J Clin Lab Analysis. 2017;31(4):e22066. PMID: 27704620. Available from: https://doi.org/10.1002/jcla 22066.

42. Joost A, Stiermaier T, Eitel C, Fuernau G, Waha S, Desch S, et al. Impact of Initial Culprit Vessel Flow on Infarct Size, Microvascular Obstruction, and Myocardial Salvage in Acute Reperfused ST-Elevation Myocardial Infarction. Am J Cardiol. 2016;118(9):1316-1322. PMID: 27600465. Available from: https://doi.org/10.1016/j.amjcard.2016.07.056.

43. Ahn SG, Hung OY, Lee JW, Lee JH, Youn YJ, Ahn MS, et al. Combination of the Thermodilution-Derived Index of Microcirculatory Resistance and Coronary Flow Reserve Is Highly Predictive of Microvascular Obstruction on Cardiac Magnetic Resonance Imaging After ST-Segment Elevation Myocardial Infarction. JACC Cardiovasc Interv. 2016;9(8):793-801. PMID: 27017370. Available from: https://doi.org/10.1016/j.jcin.2015 12.025 .

44. Zou J, Fei Q, Xiao H, Wang H, Liu K, Liu M, et al. VEGF-A promotes angiogenesis after acute myocardial infarction through increasing ROS production and enhancing ER stress-mediated autophagy. J Cell Physiol. 2019;234(10):17690-17703. PMID: 30793306. Available from: https://doi.org/10.1002/jcp.28395.

45. Douvaras P, Antonatos DG, Kekou K, Patsilinakos S, Chouliaras $\mathrm{G}$, et al. Association of VEGF gene polymorphisms with the development of heart failure in patients after myocardial infarction. Cardiology. 2009;114(1):11-18. PMID: 19332989. Available from: https://doi.org/10.1159/000210189.

46. Chin BS, Chung NA, Gibbs CR, Blann AD, Lip GY. Vascular endothelial growth factor and soluble P-selectin in acute and chronic congestive heart failure. Am J Cardiol;2002(90):12581260. Available from: https://doi.org/10.1016/S0002-9149(02) 02848-5. 